\title{
Bacillus Calmette-Guérin and anti-PD-LI combination therapy boosts immune response against bladder cancer
}

This article was published in the following Dove Press journal: OncoTargets and Therapy

\author{
Yonghua Wang' \\ Jing Liu ${ }^{2}$ \\ Xuecheng Yang' \\ Yanan Liu' \\ Yong Liu' \\ Yanjiang $\mathrm{Li}^{\prime}$ \\ Lijiang Sun' \\ Xiaokun Yang' \\ Haitao Niu' \\ 'Department of Urology, ${ }^{2}$ Department \\ of Pediatrics, The Affiliated Hospital of \\ Qingdao University, Qingdao 266000, \\ People's Republic of China
}

Background: Programmed death-ligand 1 (PD-L1) is a critical immune checkpoint molecule which promotes immunosuppression by binding to PD-1 on T-cells in tumor immunity. We have previously identified that activation of toll like receptor 4 (TLR-4), which serves an important role in the induction of antitumor immune response during Bacillus Calmette-Guérin (BCG) immunotherapy, could upregulate PD-L1 expression in bladder cancer (BCa) cells through the classical mitogen-activated protein kinase (MAPK) pathway and subsequently weaken the cytotoxicity of cytotoxic T lymphocyte (CTL). It is, therefore, necessary to investigate the possible potential relationship between PD-L1 expression and BCG immunotherapy.

Materials and methods: In this study we investigated the effects of BCG treatment on PD-L1 expression in BCa cells and also evaluated the efficacy of BCG and anti-PD-L1 combination therapy in immunocompetent orthotopic rat $\mathrm{BCa}$ models.

Results: We found that PD-L1 expression was obviously upregulated in BCa cells in response to BCG treatment both in vitro and in vivo. Moreover, BCG and anti-PD-L1 combination treatment activated a potent antitumor immune response with the increase in the number and activity of tumor-infiltrating $\mathrm{CD}^{+} \mathrm{T}$ cells, as well as the reduction in myeloid-derived suppressor cells (MDSCs), and eventually elicits prominent tumor growth inhibition and prolonged survival, and was found to be much more effective than either agent alone.

Conclusion: These findings highlight the adaptive dynamic regulation of PD-L1 in response to BCG immunotherapy and suggest that combination of BCG immunotherapy with PD-L1 blockade may be an effective antitumor strategy for improving treatment outcomes of BCa.

Keywords: BCG, PD-L1, bladder cancer, immunotherapy, combination therapy

\section{Introduction}

Bladder cancer $(\mathrm{BCa})$ is the fourth most common cancer in men in the USA, showing high morbidity and mortality rates. ${ }^{1}$ Approximately $70 \%$ of newly diagnosed $\mathrm{BCa}$ presents as non-muscle invasive bladder cancer (NMIBC). Although transurethral resection of bladder tumor (TURBT) is the primary treatment for NMIBC, recurrence rate for TURBT alone can be as high as $70 \%$, with up to $30 \%$ progressing to muscle invasive disease. ${ }^{2}$ Intravesical Bacillus Calmette-Guérin (BCG) is recommended as adjuvant therapy to reduce the risk of tumor recurrence and possibly decrease progression in intermediate and high risk NMIBC. While BCG has obtained successful responses against NMIBC, approximately $30 \%-40 \%$ of patients fail this therapy with recurrence or progression. ${ }^{3}$ The precise mechanism of BCG failure is not clear, but current studies indicate that immune resistance or immune evasion may be critical factors in the mechanisms of BCG failure., 
Programmed death-ligand 1 (PD-L1), also known as B7-H1, is a newly discovered T-cell costimulatory molecule and immune checkpoint molecule in tumor immunity. The interaction between PD-L1 and its receptor programmed death 1 (PD-1) can inhibit immune responses by inducing T-cell apoptosis, impairing cytokine production, and diminishing the cytotoxicity of activated $\mathrm{T}$ cells, and may endow tumors with a mechanism to escape host immune destruction. ${ }^{6}$ The upregulation of PD-L1 expression has been reported to be associated with poor prognosis and resistance to anticancer therapies in many malignancies, including BCa. ${ }^{7-10}$ Moreover, blockade of the PD-L1/PD-1 axis was shown to induce a potent antitumor immune response in preclinical mouse models as well as in the clinic. ${ }^{11-13}$

Our previous studies found that activation of toll like receptor 4 (TLR-4) signaling in BCa cells could upregulate PD-L1 expression through the MAPK pathway and subsequently weaken the cytotoxicity of cytotoxic T lymphocyte (CTL) against $\mathrm{BCa}$ cells. ${ }^{14} \mathrm{BCG}$ is a potent activator of TLR-4-dependent signaling and TLR-4 has also been demonstrated to serve an important role in the induction of BCG-induced antitumor immunity during intravesical BCG therapy. ${ }^{15}$ Thus, we hypothesized that BCG induces a local inflammatory response that could enhance the infiltration of tumor-specific T cells and simultaneously induce PD-L1 expression in the tumor microenvironment. The accumulation of PD-L1-expressing cells in bladder tissues may gradually impair host cell-mediated immunity, limit and even abrogate the effectiveness of BCG immunotherapy. Moreover, the concept of BCG-induced PD-L1 expression and subsequent blockade might broaden the application of PD-L1/PD-1 axis inhibitors and prove to be a potent anticancer therapy when combined with BCG immunotherapy.

In this study we first investigated the effects of BCG treatment on PD-L1 expression in BCa cell line and orthotopic rat $\mathrm{BCa}$ models. Moreover, we further evaluated whether the combination of BCG immunotherapy with PD-L1 blockade could enhance and optimize the antitumor immunity in $\mathrm{BCa}$.

\section{Materials and methods Cell culture}

Human BCa cell line T24 cells were obtained from the Cell Bank of the Chinese Academy of Sciences (Shanghai, China). Cells were cultured in RPMI 1640 media (Thermo Fisher Scientific, Waltham, MA, USA) supplemented with 10\% fetal bovine serum (Thermo Fisher Scientific), $100 \mathrm{U} / \mathrm{mL}$ penicillin (Sigma-Aldrich, St Louis, MO, USA) and $100 \mu \mathrm{g} / \mathrm{mL}$ streptomycin (Sigma-Aldrich) at $37^{\circ} \mathrm{C}$ in a humidified atmosphere containing $5 \% \mathrm{CO}_{2}$ and $95 \%$ air. Cells in the logarithmic phase of growth were used for further experiments.

\section{Establishment of orthotopic rat $\mathrm{BCa}$ model}

Female Sprague-Dawley rats (6-8 weeks of age, weighing 200-250 g) were obtained from the Experimental Animal Center of Shanghai Jiaotong University (Shanghai, China). The ethics committees from Qingdao University Affiliated Hospital have approved this animal study, and the approval was obtained prior to the commencement of the study. All animal studies were carried out following the guidelines and regulations of the Animal Care and Use Committees of Qingdao University Affiliated Hospital. All rats were housed in microisolator cages under specific pathogen-free conditions on a $12 \mathrm{~h}$ light-dark cycle. Rat BCa models were constructed by intravesical instillation of N-methyl$\mathrm{N}$-nitrosourea (MNU, Sigma-Aldrich) at $2 \mathrm{mg} /$ rat every other week for a total of four doses. At the end of the 8th week, all rats received abdominal computed tomography examination to observe the tumorigenic situation. Three rats were sacrificed and their bladders were harvested for histological study.

\section{BCG treatment}

The cultured T24 cells were pre-treated with BCG $\left(1.0 \times 10^{6}\right.$ $\mathrm{CFU} / \mathrm{mg}$, REBIO, Shanghai, China) at various concentrations (bacterium/cell ratio of 0, 2.5:1, 5:1, 10:1 and 20:1) and incubation times ( $0 \mathrm{~h}, 2 \mathrm{~h}, 4 \mathrm{~h}, 6 \mathrm{~h}, 8 \mathrm{~h}$ and $10 \mathrm{~h})$. Orthotopic tumor-bearing rats received intravesical instillation with BCG $(2.5 \mathrm{mg} / \mathrm{mL}, 0.2 \mathrm{~mL})$, or epirubicin $(5 \mathrm{mg} / \mathrm{mL}, 0.2$ $\mathrm{mL})$, or gemcitabine $(20 \mathrm{mg} / \mathrm{mL}, 0.2 \mathrm{~mL})$, or normal saline $(0.2 \mathrm{~mL})$ every week for four doses.

\section{Blockade of PD-LI with antibodies}

For the PD-L1 blockade experiment, $200 \mu \mathrm{g}$ anti-PD-L1 (clone 10F.9G2, Bio-XCell, West Lebanon, NH, USA) was administered by intraperitoneal injection to orthotopic tumor-bearing rats every 4 days for a total of seven times. For the combination of BCG with anti-PD-L1 experiment, antibody treatments were started from the day of intravesical BCG instillation.

\section{Flow cytometry analysis}

The expressions of PD-L1 in T24 cells and tumor tissues of orthotopic rat $\mathrm{BCa}$ models were evaluated by flow cytometry. Phycoerythrin-labeled PD-L1 antibody (clone MIH1/MIH5, eBioscience, San Diego, CA, USA) or isotype-matched 
control antibody was added at a concentration of $1 \mu \mathrm{g}$ per $1 \times 10^{6}$ cells per $100 \mu \mathrm{L}$ and cells were incubated for $30 \mathrm{~min}$ at $4^{\circ} \mathrm{C}$. To detect $\mathrm{CD} 8^{+} \mathrm{T}$ cells and myeloid-derived suppressor cells (MDSCs) in tumor tissues, tumor infiltrating lymphocytes (TILs) were isolated from the single cell suspension by Ficoll-Hypaque density gradient centrifugation and then stained with anti-CD8 (clone 5H10), anti-CD11b (clone M1/70) and anti-Gr1 (clone Ly-6G/Ly-6C) (eBioscience). In addition, anti-IFN- $\gamma$ (clone XMG1.2), anti-granzyme B (clone NGZB) and anti-TNF- $\alpha$ (clone MP6-XT22) (eBioscience) were used to detect IFN- $\gamma$, granzyme B and TNF- $\alpha$ secretion level in $\mathrm{CD}^{+} \mathrm{T}$ cells. Flow cytometric analysis was performed with a FACSCalibur (BD Biosciences, San Jose, CA, USA) and data were analyzed using CellQuest software (BD Biosciences).

\section{Western blot analysis}

Total proteins from tumor tissues were separated on polyacrylamide gel, transferred onto nitrocellulose membrane and incubated with anti-mouse PD-L1 antibody (clone TY25, Santa Cruz Biotechnology, Santa Cruz, CA, USA) overnight at $4^{\circ} \mathrm{C}$. Membranes were then washed three times in washing buffer and incubated with anti-rabbit IgG conjugated to peroxidase for $1 \mathrm{~h}$ at room temperature. Immunoreactivity was determined by chemiluminescence according to manufacturer instructions.

\section{Enzyme linked immunosorbent assay (ELISA)}

Serum IFN- $\gamma$ level was tested by ELISA kits (R\&D Systems, Minneapolis, MN, USA) following the manufacturer's instructions.

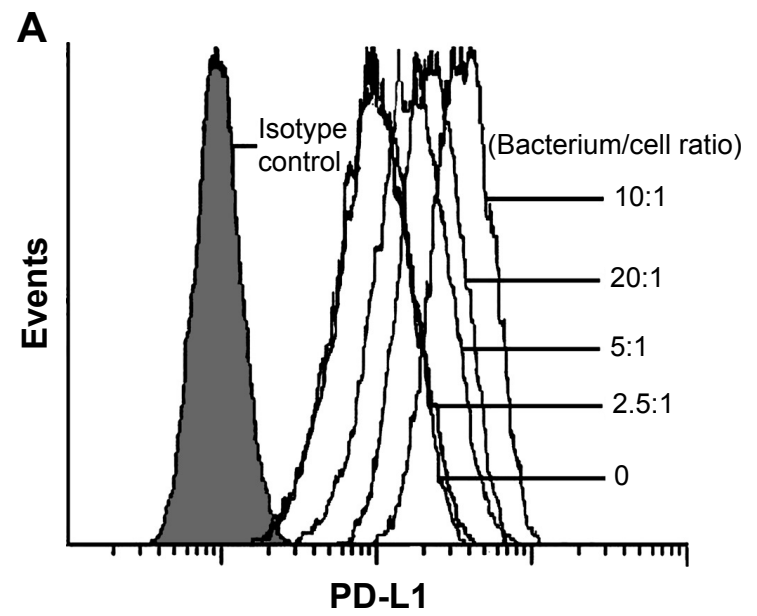

\section{Immunohistochemistry staining}

Animal tumors were sampled and embedded in paraffin. The sections were mounted on superfrost glass slides, paraffinized, rehydrated in a graded ethanol series, and then subjected to microwave antigen retrieval. Endogenous peroxidase activity was blocked by using $3 \%$ hydrogen peroxide. Sections were incubated overnight at room temperature with granzyme B antibody (catalog ab4059, Abcam, Cambridge, UK, 1:200 dilution). Staining was revealed by peroxidase/ diaminobenzidine reaction. Granzyme B stained cells were then counted and were reported in relation to the total number of cells in the tumor section.

\section{Statistical analysis}

Data were analyzed using Prism 6.0 software (GraphPad Software, San Diego, CA, USA). Experiments were repeated two or three times. Data are represented as the mean \pm standard error and the $P$-values were assessed by either Student's $t$-test or analysis of variance (ANOVA). A $P$-value $<0.05$ was considered statistically significant.

\section{Results}

\section{PD-LI expression is upregulated in T24 cells in response to $B C G$ treatment}

First, we investigated the effect of BCG treatment on PD-L1 expression in human $\mathrm{BCa}$ cell line $\mathrm{T} 24$ cells. As shown in Figure 1A, PD-L1 protein was constitutively expressed in T24 cells and was further upregulated by BCG in a concentration-dependent manner; the increases reached a peak level at a bacterium/cell ratio of 10:1. In order to determine the time course of $\mathrm{PD}-\mathrm{L} 1$ protein expression in response to BCG treatment, we treated $\mathrm{T} 24$ cells with $\mathrm{BCG}$

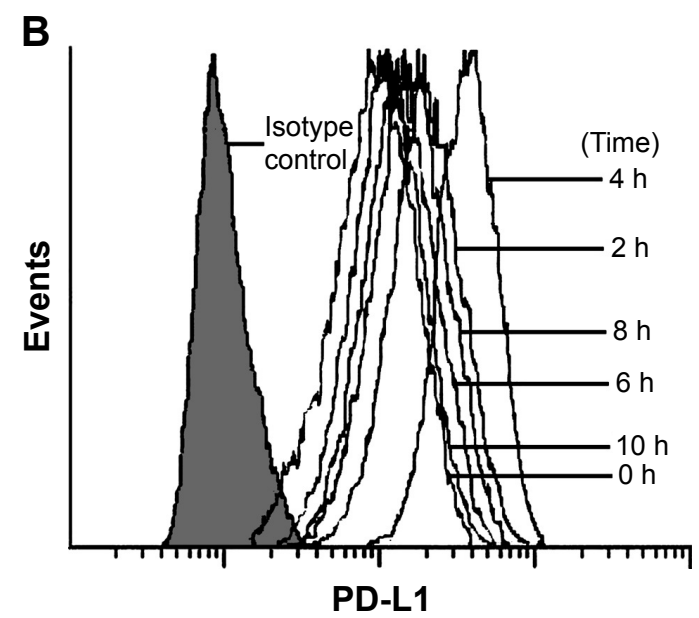

Figure I Effect of BCG treatment on PD-LI expression in T24 cells.

Notes: Concentration and time dependent increase of PD-LI protein expression by BCG treatment in T24 cells. (A) BCG (I.0XI0 $\mathrm{CFU} / \mathrm{mg})$ as bacterium/cell ratios of 0 ,

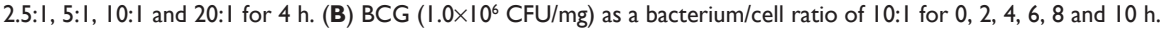

Abbreviations: BCG, Bacillus Calmette -Guérin; CFU, colony forming unit; PD-LI, programmed death-ligand I. 
(bacterium/cell ratio of 10:1) for the indicated times. Flow cytometry analysis indicated that the maximal induction of PD-L1 protein occurred at $4 \mathrm{~h}$ and then slightly decreased at 6,8 and $10 \mathrm{~h}$ (Figure 1B). These results show that BCG treatment can induce the upregulation of PD-L1 expression in $\mathrm{BCa}$ cells in vitro.

\section{Increased PD-LI expression in tumor tissue following intravesical BCG instillation}

Current animal models of BCa include chemically induced orthotopic models, xenogeneic/syngeneic transplanted models and transgenic models. To better investigate the effects of intravesical BCG immunotherapy on PD-L1 expression in vivo and exclude immunological interference factors, we established MNU-induced orthotopic rat BCa models which exhibit good tumor take, survival time, and immunocompetence. MNU-induced orthotopic rat $\mathrm{BCa}$ models were then treated with intravesical BCG instillation every week for four doses. Interestingly, we found that surface and total PD-L1 protein expressions were obviously increased in the BCG treatment group following intravesical BCG instillation by flow cytometry (Figure 2A) and Western blot (Figure 2B), respectively. In order to demonstrate that the increase in PD-L1 was not simply an effect of general treatment but unique to BCG treatment, normal saline and two other common intravesical chemotherapy agents were also used in our study as controls. Epirubicin, an anthracycline antibiotic, is a cell cycle non-specific agent that exerts antitumor effects by affecting DNA synthesis. Gemcitabine, which is a cell cycle specific agent, is an antimetabolite pyrimidine base analog that exhibits antitumor activity by inhibiting ribonucleotide reductase, incorporating into DNA and causing $\mathrm{S}$ phase arrest. However, no significant differences in PD-L1 protein expression were observed in the normal saline treatment group, epirubicin treatment group and gemcitabine treatment group. These results demonstrate that the upregulation and accumulation of PD-L1 does exist in the tumor microenvironment following intravesical BCG instillation in vivo.

\section{BCG and anti-PD-LI combination therapy enhances antitumor effects in $\mathrm{BCa}$}

As upregulation of PD-L1 has been demonstrated in $\mathrm{BCa}$ cells following BCG treatment both in vitro and in vivo, we hypothesized that blockade of PD-L1 could enhance BCG immunotherapy by alleviating the inhibitory action of PD-L1 on T cells and uncover the full cytotoxic potential of host immunity against the tumor. To test this hypothesis, MNU-induced orthotopic rat BCa models were treated with
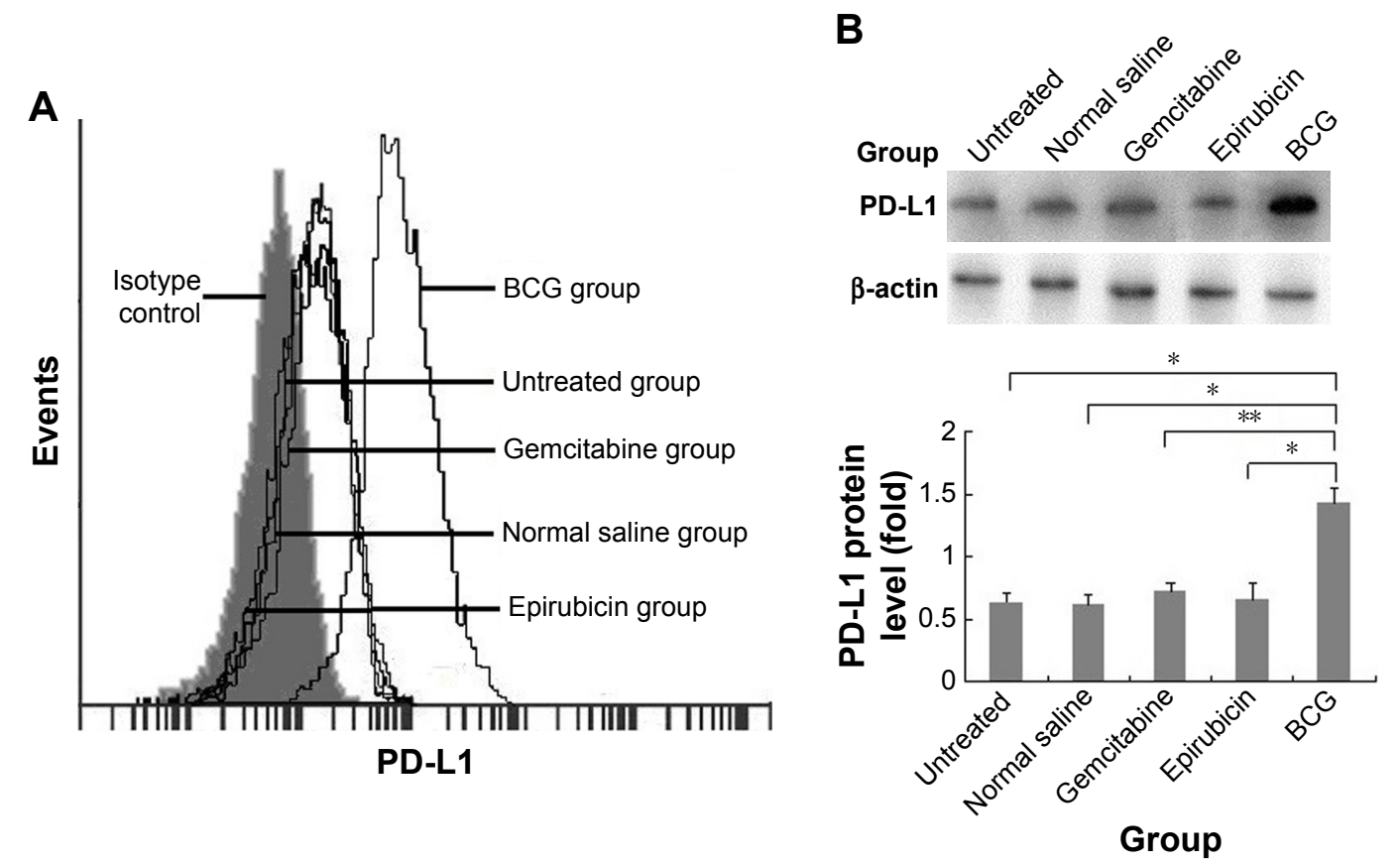

Figure 2 Effect of intravesical BCG instillation on PD-LI expression in the orthotopic rat BCa model.

Notes: PD-LI protein expressions in the tumor tissue of orthotopic rat BCa models were analyzed by flow cytometry $(\mathbf{A})$ and Western blot (B) as described in "Materials and methods" section ( $\mathrm{n}=5$ animals per group). $* \mathrm{p}<0.00 \mathrm{I} ; * * \mathrm{p}<0.01$.

Abbreviations: BCa, bladder cancer; BCG, Bacillus Calmette-Guérin; PD-LI, programmed death-ligand I. 
intravesical BCG instillation alone, anti-PD-L1 alone, or BCG plus anti-PD-L1 (Figure 3A). The results showed that with a combination treatment of BCG and anti-PD-L1 there was a significant slow-down in tumor weight as compared to single treatments or the control group (control $1.30 \pm 0.41 \mathrm{~g}$; BCG 0.86 $\pm 0.42 \mathrm{~g}$; anti-PD-L1 $0.80 \pm 0.34 \mathrm{~g}$; combination $0.35 \pm 0.14 \mathrm{~g}$ ) (Figure $3 \mathrm{~B}$ ). Moreover, survival curves showed that combination treatment of BCG and anti-PD-L1 did significantly prolong survival over single treatments or the control group (Figure 3C). Thus, these results demonstrated that the combination therapy was superior to monotherapy with either agent in exerting an antitumor response.

\section{BCG and anti-PD-LI combination therapy boosts antitumor immunity in $\mathrm{BCa}$}

We next examined whether combination therapy could activate adaptive immune responses. We analyzed the percentage of tumor-infiltrating $\mathrm{CD}^{+} \mathrm{T}$ cells by flow cytometry and found that tumor-infiltrating $\mathrm{CD}^{+} \mathrm{T}$ cells were increased by BCG and/or anti-PD-L1 treatment (Figure 4A). Interestingly, BCG and anti-PD-L1 combination therapy induced the greatest increase in tumor-infiltrating $\mathrm{CD}^{+} \mathrm{T}$ cells, as compared to single treatments or the control group. In parallel, significant increases in the percentage of $\mathrm{CD}^{+}$ T cells producing granzyme B, IFN- $\gamma$ and TNF- $\alpha$ were also observed in the combination treatment group compared to single treatments or the control group, indicating that BCG and anti-PD-L1 combination treatment not only elevated the number of tumor-infiltrating $\mathrm{CD} 8^{+} \mathrm{T}$ cells, but also strengthened their antitumor activity (Figure 4B and 4C). In addition, serum IFN- $\gamma$ level was also further elevated in the combination treatment group, demonstrating that BCG and anti-PD-L1 combination therapy significantly strengthened immune response in $\mathrm{BCa}$ (Figure 4D).

MDSCs in the tumor microenvironment can suppress immune responses and facilitate tumor relapse. To determine the dynamics of MDSCs accumulation in the tumor microenvironment, we sought to investigate possible changes in the percentage of MDSCs following treatment. We observed that MDSCs, defined by $\mathrm{CD} 45^{+} \mathrm{CD} 11 \mathrm{~b}^{+} \mathrm{Gr} 1^{+}$expression, were reduced by $\mathrm{BCG}$ and/or anti-PD-L1 treatment. In tumors that received $\mathrm{BCG}$ or anti-PD-L1 treatment alone, the percentage of MDSCs decreased from $12.04 \% \pm 1.03 \%$ in untreated

A

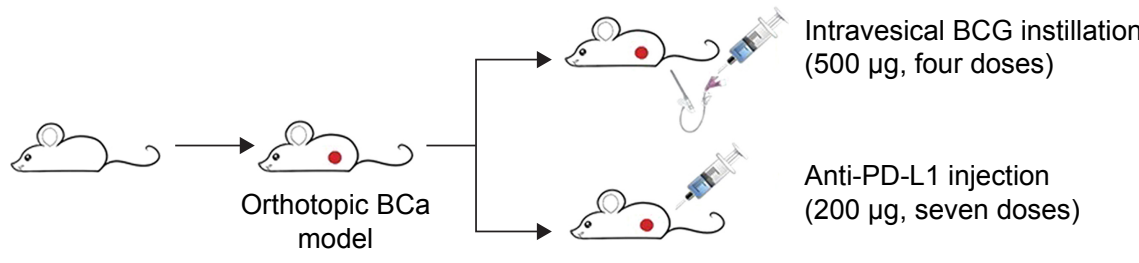

B

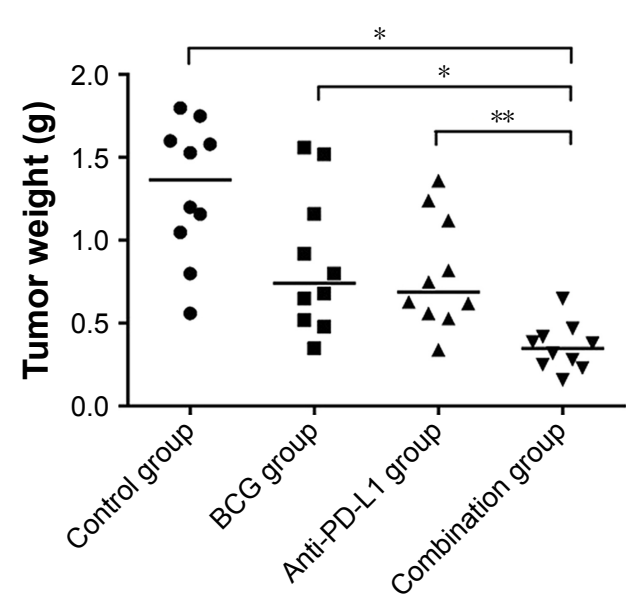

C

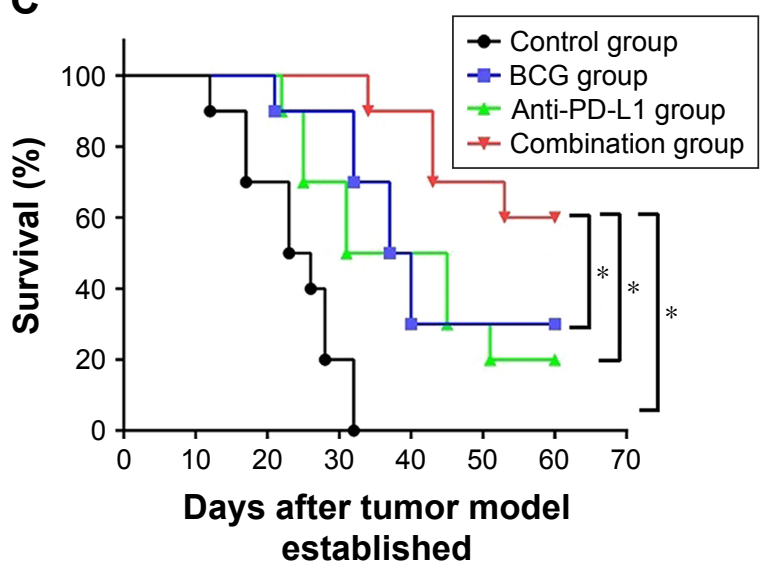

Figure 3 BCG and anti-PD-LI combination therapy inhibited tumor progression and prolonged survival in the orthotopic rat BCa model.

Notes: (A) Study design: orthotopic rat BCa models received intravesical BCG instillation $(500 \mu \mathrm{g})$ every week for four doses and/or $200 \mu \mathrm{g}$ anti-PD-LI (clone I0F.9G2) or isotype control i.p. every 4 days for a total of seven times ( $n=10$ animals per group). Tumor weight (B) and survival percentage (C) were monitored and measured at the endpoint (animal death date or day 60). $* P<0.01 ; * * P<0.05$.

Abbreviations: BCG, Bacillus Calmette-Guérin; i.p., intraperitoneal injection; PD-LI, programmed death-ligand I. 


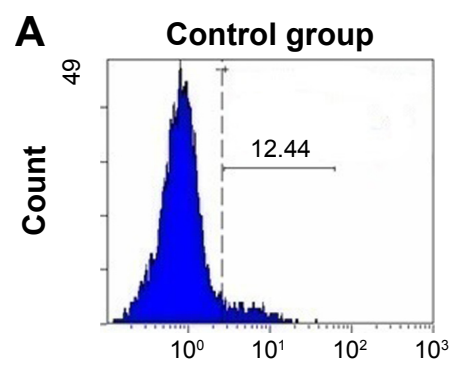

FL1 log: CD8 FITC

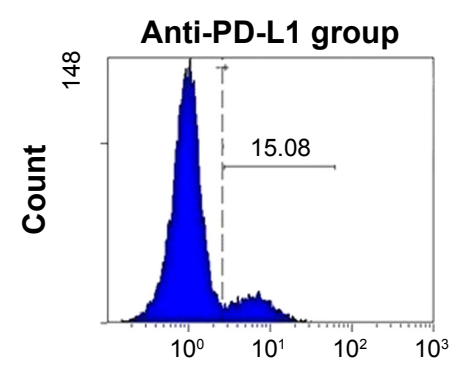

FL1 log: CD8 FITC

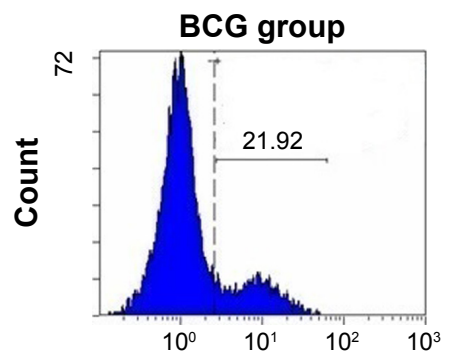

FL1 log: CD8 FITC

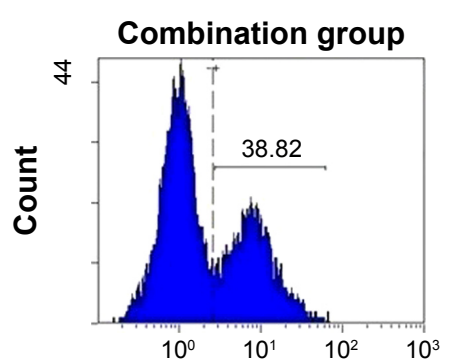

FL1 log: CD8 FITC

B

Control group

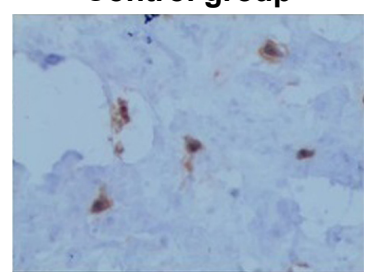

Anti-PD-L1 group

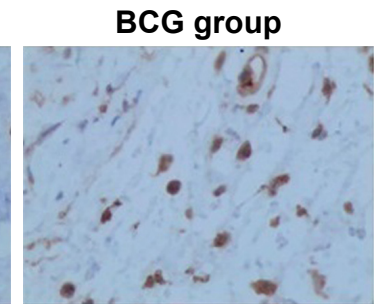

Combination group
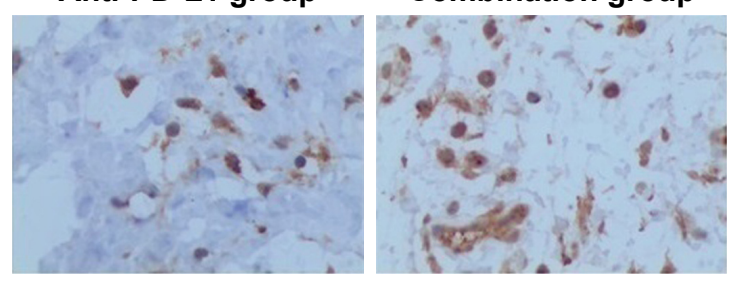

C

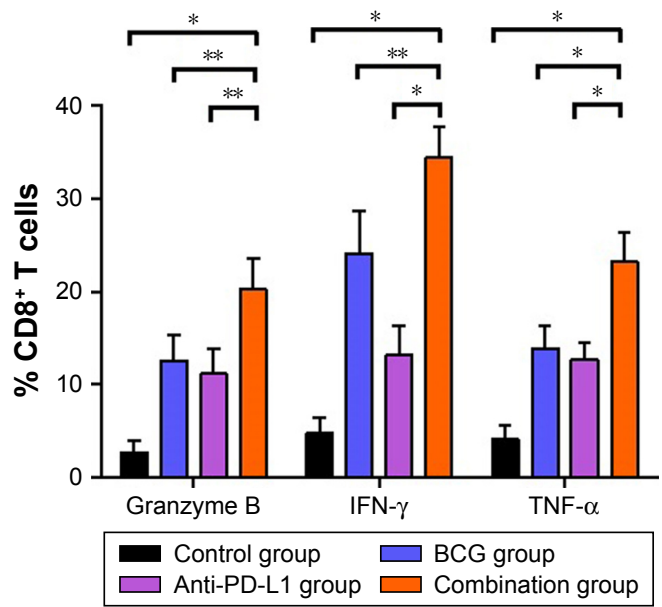

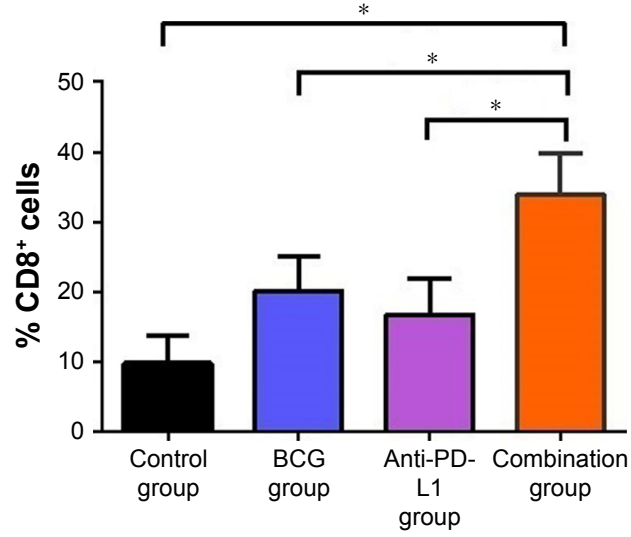

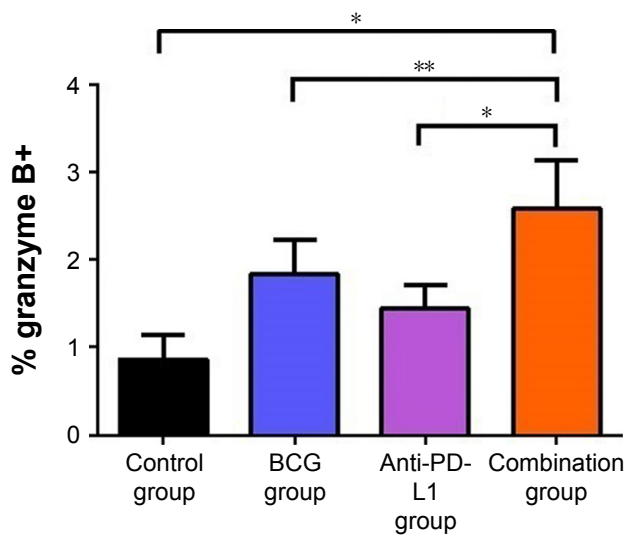

D

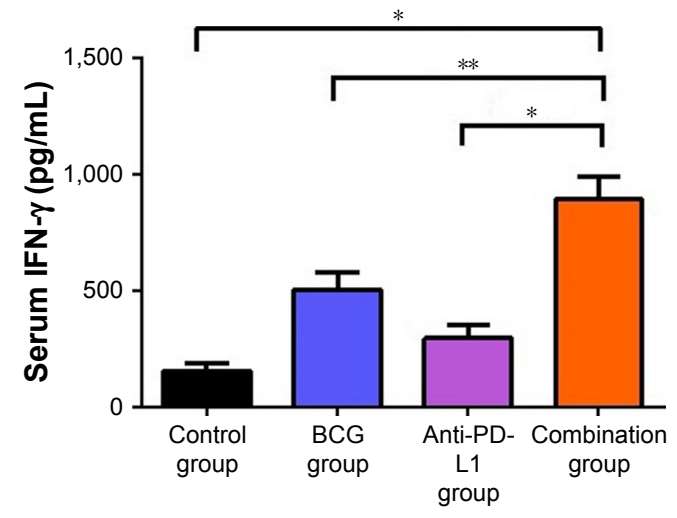

Figure 4 BCG and anti-PD-LI combination therapy elevated the number and activity of tumor-infiltrating CD8 ${ }^{+} \mathrm{T}$ cells in the orthotopic rat BCa model.

Notes: (A) Left panel: representative histograms obtained by flow cytometry showing the fluorescence intensity of CD8 $8^{+} \mathrm{T}$ cells. Right panel: quantitative data of tumorinfiltrating CD8 ${ }^{+} \mathrm{T}$ cells from different groups is shown. (B) Left panel: representative images showing immunostaining for granzyme B (brown staining) in tumors from different groups. Right panel: quantification of the percentage of granzyme $B$ positive cells on tumor sections. (C) Representative data showed the percentage of $C D 8^{+} T$ cells producing granzyme B, IFN- $\gamma$ and TNF- $\alpha$ from different groups by flow cytometry. (D) Serum IFN- $\gamma$ level from different groups, given by ELISA assay. $* P<0.00$ I; $* * P<0.0$ I. Abbreviations: BCa, bladder cancer; BCG, Bacillus Calmette-Guérin; ELISA, enzyme linked immunosorbent assay; FITC, fluorescein isothiocyanate; PD-LI, programmed death-ligand I. 


\section{Control group}

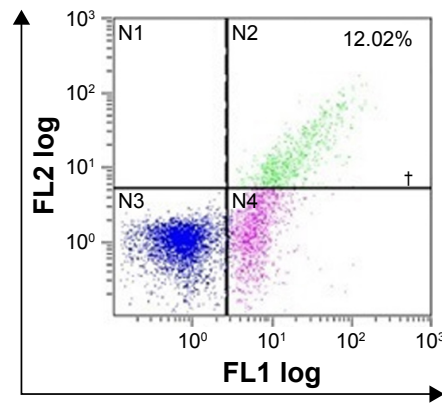

\section{FL1 log (FITC-CD11b+)l} FL2 log (PE-Gr1+)

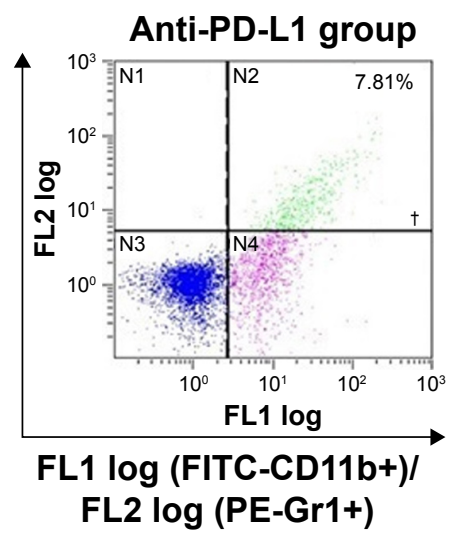

BCG group

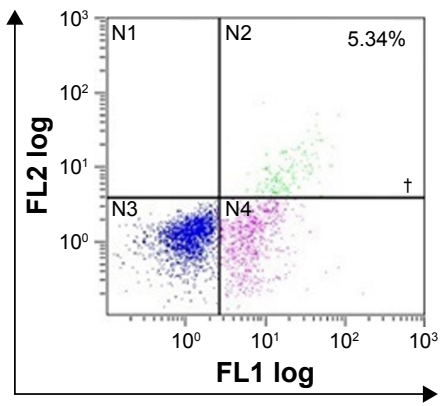

FL1 log (FITC-CD11b+)/ FL2 log (PE-Gr1+)

\section{Combination}

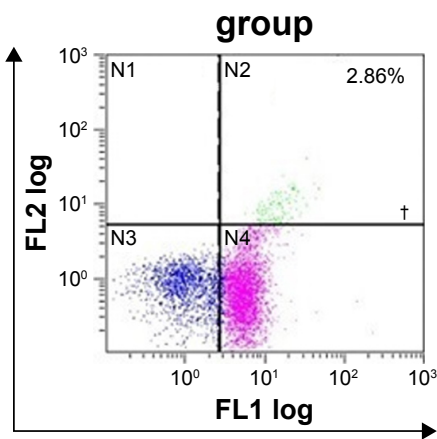

FL1 log (FITC-CD11b+)/ FL2 log (PE-Gr1+)

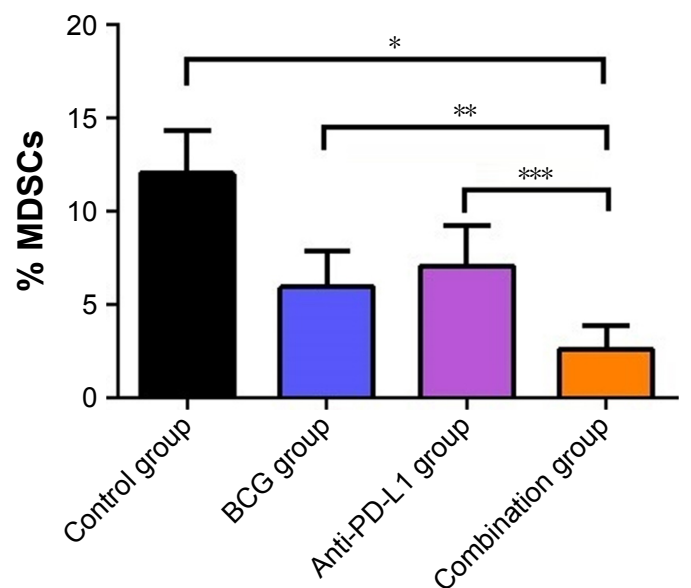

Figure 5 BCG and anti-PD-LI combination therapy reduced the number of MDSCs in the orthotopic rat BCa model.

Notes: Left panel: representative dot plots obtained by flow cytometry showing the fluorescence intensity of MDSCs. Right panel: quantitative data of the percentage of MDSCs from different groups is shown. $* P<0.001$; $* * P<0.05$; $* * * P<0.01$.

Abbreviations: BCa, bladder cancer; BCG, Bacillus Calmette-Guérin; FITC, fluorescein isothiocyanate; MDSCs, myeloid-derived suppressor cells; PD-LI, programmed death-ligand I; PE, phycoerythrin.

tumors to $5.96 \% \pm 0.88 \%$ and $7.09 \% \pm 0.97 \%$, respectively. Combination treatment of BCG and anti-PD-L1 exhibited the greatest effect on MDSCs and further reduced the percentage to $2.65 \% \pm 0.55 \%$ (Figure 5). Our results indicated that BCG and anti-PD-L1 combination therapy successfully boosted host antitumor immunity, not only by enhancing $\mathrm{T}$ cell immune response but also by reducing the accumulation of MDSCs in the tumor microenvironment.

\section{Discussion}

Current studies indicate that immune resistance factors play important roles in the mechanism of BCG failure, which is one of major obstacles in the treatment of NMIBC. PD-L1, as a vital inhibitory checkpoint molecule, has gained increasing attention in tumor immunity. PD-L1 expression on tumor cells has been linked to a weakened host immune response, consequent poor prognosis and resistance to anti-cancer therapies in various malignancies, including lung, breast, gastric, colorectal, ovarian, and renal cancers, and $\mathrm{BCa} .^{7-10}$ Although previous studies have pointed out the possibility that BCG treatment may lead to the upregulation of PD-L1 expression as well as inducing a strong local inflammatory response, up to now little is known about the detailed relationship between PD-L1 expression and BCG immunotherapy in $\mathrm{BCa}$, which needs to be clarified.

In the present study, we investigated the effect of BCG treatment on $\mathrm{PD}-\mathrm{L} 1$ expression in $\mathrm{BCa}$ cells and also evaluated the efficacy of BCG and anti-PD-L1 combination therapy in immunocompetent orthotopic rat $\mathrm{BCa}$ models. We confirmed that PD-L1 expression was obviously upregulated in $\mathrm{BCa}$ cells in response to BCG treatment both in vitro and in vivo. Furthermore, we demonstrated that BCG and antiPD-L1 combination therapy exerted an enhanced antitumor effect on orthotopic rat $\mathrm{BCa}$ models by reducing tumor burden and prolonging survival. The antitumor immunity also got a boost with an obvious increase in the number and activity of tumor-infiltrating $\mathrm{CD}^{+} \mathrm{T}$ cells, as well as suppression of MDSCs in the tumor microenvironment.

The relationship between PD-L1 expression and BCG induction in the inflammatory immune response has been 
studied; eg, Gouveia et al described that an enhanced expression of PD-L1 was observed on dendritic cells in a mouse model of airway inflammation after neonatal BCG vaccination. ${ }^{16}$ Zhou et al demonstrated that mycobacterium tuberculosis infection could induce increased expression of PD-L1 in macrophage by Akt-mTORC1 pathway. ${ }^{17}$ Recently, Inman et al found that 11 of 12 patients with BCa who failed BCG immunotherapy exhibited extremely intense PD-L1 expression within the BCG granulomas of tumor tissue. ${ }^{18}$ Heo et al also showed that normal epithelial tissues regenerated after surgical resection and BCG immunotherapy, had low expression of PD-L1, while recurred tumor showed high expression of PD-L1 after surgical resection and BCG immunotherapy. ${ }^{19}$ These clinical findings support the observations in our study and suggest that further research is needed to demonstrate the predictive role of PD-L1 expression in the effectiveness of $\mathrm{BCG}$ immunotherapy.

After four decades of the successful application of BCG immunotherapy in NMIBC, anti-PD-L1 therapy has already shown great promise as a cancer immunotherapy for advanced $\mathrm{BCa}$ in recent years. The success and limitations of both BCG immunotherapy and current anti-PD-L1 therapy have inspired multiple combination strategies to improve their therapeutic efficacy and clinical response. In the present study our results demonstrated that BCG and anti-PD-L1 combination therapy elicited a potent antitumor immune response with much more increase in tumor-infiltrating $\mathrm{CD}^{+}$ $\mathrm{T}$ cells than either agent alone. Moreover, strong increases in the expression of granzyme B, IFN- $\gamma$ and TNF- $\alpha$ on $\mathrm{CD}^{+} \mathrm{T}$ cells were found in the tumors treated with $\mathrm{BCG}$ and anti-PD-L1 combination therapy. Granzyme B, IFN- $\gamma$ and TNF- $\alpha$ are all known to be released by cytotoxic T cells and symbolize a cytotoxic $\mathrm{T}$ cell immune response. ${ }^{20-22}$ Thus, these data reflect an enhancement in the killing abilities of tumor-infiltrating $\mathrm{CD}^{+} \mathrm{T}$ cells in the tumor microenvironment. In addition, high IFN- $\gamma$ levels were also detected in the serum of rats treated with combination therapy. All these findings indicate that BCG and anti-PD-L1 combination therapy can activate the adaptive immune system to exert a cytotoxic immune response.

It has been shown that MDSCs have a close relationship with PD-L1/PD-1 axis and BCG immunotherapy. ${ }^{23,24}$ It has been demonstrated that MDSCs could upregulate PD-1 expression in $\mathrm{CD}^{+} \mathrm{T}$ cells and induce activated $\mathrm{T}$ cell exhaustion, ${ }^{23}$ and in addition, recent studies indicated that $\mathrm{CD} 8^{+} \mathrm{T}$ cell-toMDSC balance was associated with the recurrence of $\mathrm{BCa}$ undergoing $\mathrm{BCG}$ immunotherapy. ${ }^{24}$ Patients with a low
$\mathrm{CD}^{+} \mathrm{T}$ cell/MDSC ratio showed high recurrence rate after intravesical BCG immunotherapy. In our study we found that MDSCs in tumor tissues were reduced by BCG or anti-PD-L1 therapy, while the combination therapy exhibited the greatest inhibitory effect. Previous studies have demonstrated that activated $\mathrm{CD}^{+} \mathrm{T}$ cells could induce an increase in MDSCs apoptosis in a TNF- $\alpha$-dependent manner. ${ }^{25}$ Indeed, we also found the expression of TNF- $\alpha$ on $\mathrm{CD}^{+} \mathrm{T}$ cells was significantly increased in the tumors treated with combination therapy. Thus, we speculate that our combination therapy could enhance cytotoxic $\mathrm{T}$ cell functions, which, in turn, negatively regulate the accumulation of MDSCs. Further studies are still needed to clarify the precise mechanisms of MDSCs downregulation with BCG and anti-PD-L1 combination therapy.

Several ongoing clinical trials are investigating the rational combination of BCG therapy with PD-L1/PD-1 blockade. NCT02792192 is a Phase Ib/II clinical study assessing combination atezolizumab (anti-PD-L1 antibody) with BCG therapy for high-risk NMIBC. NCT02324582 and NCT02808143 are both Phase I clinical studies assessing pembrolizumab (anti-PD-1 antibody) with BCG therapy for high-risk or BCG-refractory NMIBC. Our findings may provide a preliminary theoretical basis and support for these clinical trials, which will provide more implications on the efficacy and tolerability of the combination therapy and, hopefully, better outcomes for patients with $\mathrm{BCa}$.

There are several limitations to the present study. First, our study is limited to one kind of BCa cell and animal model. The sample size of the animal model is relatively small. Second, our study mainly focused on $\mathrm{CD}^{+} \mathrm{T}$ cells and MDSCs in the immune response with combination therapy, while some other immune cell subsets, such as $\mathrm{CD}^{+}{ }^{+} \mathrm{T}$ cells, NK cells, neutrophils, monocytes and Tregs, may also play roles in this process. Thus, more tumor cell lines, animal models and involved immune cells are warranted to verify our findings in further studies. In addition, precise mechanisms such as PD-L1 upregulation by BCG and the synergy effect between BCG and anti-PD-L1 therapy need to be well studied in the future.

In conclusion, this is the first study demonstrating that BCG treatment can induce increased expression of PD-L1 in BCa cells. BCG and anti-PD-L1 combination therapy can boost immune response by stimulating $\mathrm{CD} 8^{+} \mathrm{T}$ cell activity and suppressing MDSCs, and eventually elicit a potent antitumor effect against $\mathrm{BCa}$. Our results suggest that BCG and anti-PD-L1 combination therapy may be a promising therapeutic strategy for the treatment of $\mathrm{BCa}$ patients. 


\section{Acknowledgments}

This work was supported by National Natural Science Foundation of China (81101932); Science and Technology Project of Qingdao (15-9-1-105-jch); and Higher Educational Science and Technology Program of Shandong (J16LL04).

\section{Disclosure}

The authors report no conflicts of interest in this work.

\section{References}

1. Siegel RL, Miller KD, Jemal A. Cancer Statistics, 2017. CA Cancer J Clin. 2017;67(1):7-30.

2. Babjuk M, Böhle A, Burger M, et al. EAU Guidelines on Non-Muscleinvasive Urothelial Carcinoma of the Bladder: Update 2016. Eur Urol 2017;71(3):447-461.

3. Yates DR, Brausi MA, Catto JW, et al. Treatment options available for bacillus Calmette-Guérin failure in non-muscle-invasive bladder cancer. Eur Urol. 2012;62(6):1088-1096.

4. Wu Y, Enting D, Rudman S, Chowdhury S. Immunotherapy for urothelial cancer: from BCG to checkpoint inhibitors and beyond. Expert Rev Anticancer Ther. 2015;15(5):509-523.

5. Wang Y, Yang M, Yu Q, Yu L, Shao S, Wang X. Recombinant bacillus Calmette-Guérin in urothelial bladder cancer immunotherapy: current strategies. Expert Rev Anticancer Ther. 2015;15(1):85-93.

6. Tumeh PC, Harview CL, Yearley JH, et al. PD-1 blockade induces responses by inhibiting adaptive immune resistance. Nature. 2014; 515(7528):568-571.

7. Gubin MM, Zhang X, Schuster H, et al. Checkpoint blockade cancer immunotherapy targets tumour-specific mutant antigens. Nature. 2014;515(7528):577-581.

8. Wang X, Teng F, Kong L, Yu J. PD-L1 expression in human cancers and its association with clinical outcomes. Onco Targets Ther. 2016;9: 5023-5039.

9. Balar AV, Weber JS. PD-1 and PD-L1 antibodies in cancer: current status and future directions. Cancer Immunol Immunother. 2017;66(5): $551-564$

10. Yu L, Wang Y, Shao S, et al. B7-H1/PD-1 blockade therapy in urological malignancies: current status and future prospects. Tumori. 2015;101(5) 549-554.

11. Topalian SL, Hodi FS, Brahmer JR, et al. Safety, activity, and immune correlates of anti-PD-1 antibody in cancer. N Engl J Med. 2012;366(26): 2443-2454.
12. Sznol M, Chen L. Antagonist antibodies to PD-1 and B7-H1 (PD-L1) in the treatment of advanced human cancer. Clin Cancer Res. 2013;19(5): 1021-1034.

13. Rijnders M, de Wit R, Boormans JL, Lolkema MPJ, van der Veldt AAM. Systematic review of immune checkpoint inhibition in urological cancers. Eur Urol. 2017;72(3):411-423.

14. Wang YH, Cao YW, Yang XC, et al. Effect of TLR4 and B7-H1 on immune escape of urothelial bladder cancer and its clinical significance. Asian Pac J Cancer Prev. 2014;15(3):1321-1326.

15. Redelman-Sidi G, Glickman MS, Bochner BH. The mechanism of action of BCG therapy for bladder cancer - a current perspective. Nat Rev Urol. 2014;11(3):153-162.

16. Gouveia ACC, Braga FG, Mota M, et al. Enhanced expression of PD-L1 and IFN- $\gamma$ on dendritic cells is associated with BCG-induced Th2 inhibition. Cytokine. 2017;99:163-172.

17. Zhou Y, Hu Z, Cao S, Yan B, Qian J, Zhong H. Concomitant Mycobacterium tuberculosis infection promotes lung tumor growth through enhancing Treg development. Oncol Rep. 2017;38(2):685-692.

18. Inman BA, Sebo TJ, Frigola X, et al. PD-L1 (B7-H1) expression by urothelial carcinoma of the bladder and BCG-induced granulomata: associations with localized stage progression. Cancer. 2007;109(8): 1499-1505.

19. Heo JH, Jin HA, Kang YH, Kim KH, Hong SJ, Han KS. Expression of PD-L1 and BCG immunotherapy in non-muscle invasive bladder cancer. Cancer Res. 2016;76(15S):A16. Abstract.

20. Shao Y, Zhu W, Da J, et al. Bisdemethoxycurcumin in combination with $\alpha$-PD-L1 antibody boosts immune response against bladder cancer. Onco Targets Ther. 2017;10:2675-2683.

21. Curran MA, Montalvo W, Yagita H, Allison JP. PD-1 and CTLA-4 combination blockade expands infiltrating $\mathrm{T}$ cells and reduces regulatory $\mathrm{T}$ and myeloid cells within B16 melanoma tumors. Proc Natl Acad Sci U S A. 2010;107(9):4275-4280.

22. Nakhlé J, Pierron V, Bauchet AL, et al. Tasquinimod modulates tumor-infiltrating myeloid cells and improves the antitumor immune response to PD-L1 blockade in bladder cancer. Oncoimmunology. 2016;5(6):e1145333.

23. Zhu H, Gu Y, Xue Y, Yuan M, Cao X, Liu Q. CXCR2+ MDSCs promote breast cancer progression by inducing EMT and activated $\mathrm{T}$ cell exhaustion. Oncotarget. 2017;8(70):114554-114567.

24. Chevalier MF, Trabanelli S, Racle J, et al. ILC2-modulated T cell-toMDSC balance is associated with bladder cancer recurrence. $J$ Clin Invest. 2017;127(8):2916-2929.

25. Deng L, Liang H, Burnette B, et al. Irradiation and anti-PD-L1 treatment synergistically promote antitumor immunity in mice. JClin Invest. 2014;124(2):687-695.
OncoTargets and Therapy

\section{Publish your work in this journal}

OncoTargets and Therapy is an international, peer-reviewed, open access journal focusing on the pathological basis of all cancers, potential targets for therapy and treatment protocols employed to improve the management of cancer patients. The journal also focuses on the impact of management programs and new therapeutic agents and protocols on

\section{Dovepress}

patient perspectives such as quality of life, adherence and satisfaction. The manuscript management system is completely online and includes a very quick and fair peer-review system, which is all easy to use. Visit http://www.dovepress.com/testimonials.php to read real quotes from published authors. 